\title{
Complicações per e pós-operatórias em 1000 olfios submetidos a LASIK
}

An analysis of intraoperative and postoperative complications in 1000 laser in situ
Keratomileusis
cases

$\begin{array}{lll}\text { Telma } & \text { Pereira }^{1-2} & \\ \text { Adriana } & \text { dos Santos } & \text { Forseto }^{1-3} \\ \text { Walton } & \text { Nosé }^{1-3} & \end{array}$

\begin{tabular}{|c|}
\hline RESUMO \\
\hline $\begin{array}{l}\text { Objetivo: Analisar a incidência, tipo, tratamento e evolução das compli- } \\
\text { cações em LASIK. Métodos: Estudo retrospectivo de } 1000 \text { olhos subme- } \\
\text { tidos a LASIK usando os microceratótomos Hansatome }{ }^{\circledR} \text { ou Automated } \\
\text { Corneal Shaper }{ }^{\circledR} \text { e os aparelhos de Excimer Laser VISX } 20 / 20 \text { B ou Chiron } \\
\text { Technolas } 217 \text { C. Complicações peroperatórias e pós-operatórias preco- } \\
\text { ces e tardias foram analisadas. Resultados: A média do equivalente } \\
\text { esférico pré-operatório foi de - } 4,29 \pm 3,20 D \text {. A média do seguimento foi } \\
\text { de } 6,05 \pm 6,69 \text { meses. No período peroperatório foram encontradas cinco } \\
(0,5 \%) \text { complicações do disco relacionadas ao microceratótomo ( } 3 \text { discos } \\
\text { finos, } 1 \text { disco com perfuração central e } 1 \text { disco pequeno). As complica- } \\
\text { ções mais freqüentes no período pós-operatório precoce foram as dobras } \\
\text { de disco }(6,4 \%) \text {, seguidas de debris na interface }(4,1 \%) \text {, ceratite não } \\
\text { específica da interface (1,1\%), "haze" ( } 0,4 \%) \text {, crescimento epitelial da } \\
\text { interface }(0,4 \%) \text { e deslocamento de disco ( } 0,3 \%) \text {. A maioria destes } \\
\text { eventos foi prontamente tratada, alcançando-se bons resultados. Para as } \\
\text { complicações tardias, relacionadas à refração, foram analisados } 655 \\
\text { olhos que apresentavam seguimento mínimo de } 3 \text { meses. No último } \\
\text { exame, a média do equivalente esférico neste grupo foi de - } 0,26 \pm 0,76 D \text {. } \\
\text { Oito por cento dos olhos encontravam-se com hipocorreção superior a } \\
1,00 D ; \text {; } 1,67 \% \text { com hipercorreção }>1,00 D \text {. Retratamento foi necessário } \\
\text { em } 28 \text { olhos ( } 4,27 \%) \text {. Cinco casos }(0,76 \%) \text { perderam de } 2 \text { ou mais linhas } \\
\text { da melhor acuidade visual corrigida. Não foram observadas outras } \\
\text { complicações visualmente importantes. Conclusão: LASIK é um proce- } \\
\text { dimento refrativo seguro e com poucas complicacões. }\end{array}$ \\
\hline
\end{tabular}

Descritores: Ceratomileuse assistida por excimer laser in situ/efeitos adversos; Ceratomileuse assistida por excimer laser in situ/métodos; Erros de refração/ cirurgia; Estudos retrospectivos; Complicações intra-operatorias/epidemiologia; Complicações pós-operatorias/epidemiologia

Trabalho realizado no Eye Clinic Day Hospital - São Paulo (SP).

${ }^{1}$ Eye Clinic Day Hospital (SP)

${ }^{2}$ Faculdade de Medicina da Fundação do ABC

${ }^{3}$ Universidade Federal de São Paulo - Escola Paulista de Medicina (UNIFESP/EPM).

Endereço para correspondência: Av. República do Líbano, 1034 - São Paulo (SP) CEP 04502-001. E-mail: wnose@uol.com.br

\section{INTRODUÇÃO}

A cirurgia refrativa apresentou desenvolvimento extraordinário com o advento do Excimer Laser, equipamento capaz de remover quantidade precisa de tecido corneano através da fotoablação ${ }^{(1)}$.

Inicialmente aplicado na superfície da córnea, após a remoção do epitélio (PRK ou Ceratectomia Fotorrefrativa) ${ }^{(2)}$ o Excimer Laser logo foi associado à já conhecida técnica de cirurgia lamelar a Ceratomileusis, surgindo assim o LASIK (Laser in Situ Keratomileusis). 
A atual técnica de $\operatorname{LASIK}^{(3)}$, graças aos bons resultados refracionais obtidos, vem sendo empregada de forma crescente para correção dos mais variados erros refrativos, devido em parte a avanços técnicos dos equipamentos de Excimer Laser e dos microceratótomos.

A recuperação visual mais precoce ${ }^{(4)}$ e o maior conforto pós-operatório ${ }^{(4-5)}$ tornaram a técnica de LASIK mais popular quando comparada à técnica de $\mathrm{PRK}^{(4)}$.

Entretanto, a curva de aprendizado do LASIK é considerada mais difícil, o que pode estar relacionado a maior incidência de complicações, até que haja um domínio da técnica ${ }^{(6)}$.

\section{MÉTODOS}

Foram analisados retrospectivamente os prontuários de 531 pacientes (1000 olhos) submetidos a LASIK entre junho de 1996 e junho de 2000, com o objetivo de avaliar a incidência, tipo e evolução de complicações.

Foram coletados os dados refracionais do período pré e pós-operatórios medidos da acuidade visual com e sem correção, tipo de microceratótomo e aparelho de excimer laser utilizado, assim como a presença de complicações no período per-operatório, pós-operatório precoce e tardio.

A técnica de LASIK já foi aprovada para uso clínico no Brasil pelo Conselho Federal de Medicina e Conselho Brasileiro de Oftalmologia. Desta forma, obteve-se termo de consentimento assinado em todos os casos, após explicação detalhada dos procedimentos cirúrgicos, seus riscos e outras opções para a correção de suas ametropias.

A avaliação no período pré-operatório consistiu de exame oftalmológico completo (AV s/c e c/c, refratometria dinâmica e estática, biomicroscopia à lâmpada de fenda, tonometria e mapeamento de retina), e ainda topografia computadorizada da córnea (Eye Map EH-290, Alcon, Irvine, Califórnia); ou Eye Sys, Eye Sys, Houston, Texas) e paquimetria ultrassônica (Ultrasonic Pachymeter Paradigm Medical Industries, Salt Lake City, Utah). Os pacientes foram orientados a suspender o uso de suas lentes de contato por período mínimo de 1 semana ou mais, dependendo do tipo de lente utilizada ou da presença de alterações topográficas sugestivas de "warpage" corneano. Foram excluídos pacientes com alterações topográficas sugestivas de doenças ectásicas da córnea, glaucoma, ou outras doenças oculares.

Todas as cirurgias foram realizadas sob anestesia tópica (cloridrato de proximetacaína, Anestalcon ${ }^{\circledR}$ ), pelo mesmo cirurgião (WN). Resumidamente, a preparação do disco corneano e leito estromal no LASIK foi realizada com os microceratótomos Automated Corneal Shaper - ACS (Chiron Ophthalmics $^{\circledR}$ ), com plataformas de 130 ou $160 \mu$ m e diâmetro de $8,5 \mathrm{~mm}$; ou Hansatome (Bausch \& Lomb Surgical ${ }^{\oplus}$ ), plataformas de 160 ou $180 \mu \mathrm{m}$, e diâmetro de 8,5 ou $9,5 \mathrm{~mm}$. A fotoablação corneana foi feita com os aparelhos de excimer laser VISX 20/20B (versão $4,02 \mathrm{c})(\mathrm{n}=410)$, que trabalha com fluência de $160 \mathrm{~mJ} / \mathrm{cm}^{2} \mathrm{e}$ taxa de repetição de $6 \mathrm{~Hz}$; ou Chiron-Technolas $217 \mathrm{C}$ (Bausch
\& Lomb Surgical ${ }^{\circledast}$, versão 2 ,9992) $(\mathrm{n}=590)$, que opera com sistema denominado PlanoScan e taxa de repetição de $50 \mathrm{~Hz}$. No final do procedimento, o disco foi reposicionado sem a necessidade de oclusão ou lentes de contato terapêuticas, tendo sido prescrito colírio de tobramicina $0,3 \%$ e dexametasona $0,1 \%$ (Tobradex $^{\circledR}: 4 / 4 \mathrm{~h}$ por 10 dias).

As visitas no período pós-operatório foram agendadas para $1,3,5$ e 15 dias e 1, 3, 6, 12 e 24 meses, quando foram realizados exames oftalmológicos completos.

RESULTADOS

Os resultados apresentados referem-se aos dados de 1000 olhos de 531 pacientes, com idade média de 33,25 $\pm 8,75$ anos (variando de 18 a 64 anos). Destes 531 pacientes, 257 (48,39\%) eram do sexo feminino. Não houve diferença em relação ao lado operado e o sexo. Temos que o lado direito $51,30 \%$ eram homens e $48,70 \%$ eram mulheres e o lado esquerdo $52,89 \%$ eram homens e $47,11 \%$ eram mulheres (teste Chi-Square $\mathrm{p}=0,614$ ).

A média de seguimento foi de 6,05 $\pm 6,69$ meses (variando de 1 a 50 meses). Vinte e sete olhos $(0,27 \%)$ haviam sido submetidos à cirurgia ocular prévia: facoemulsificação com implante de lente intra-ocular $(\mathrm{n}=4)$; transplante de córnea $(\mathrm{n}=7)$; cirurgia de estrabismo $(\mathrm{n}=1)$; ceratotomia radial $(\mathrm{n}=8)$; ceratectomia fotorrefrativa $(n=5)$; e LASIK $(n=2)$. O tempo médio de realização destas cirurgias antes do LASIK foi de $3,48 \pm 2,31$ anos (variando de 1 a 11 anos; a variância do tempo da cirurgia é de 5,34). Quatro dos 1000 olhos $(0,4 \%)$ foram submetidos a tratamento profilático no período pré-operatório com fotocoagulação por laser de argônio devido a degenerações retinianas pela alta miopia.

Setecentos e noventa e quatro olhos $(79,4 \%)$ apresentavam acuidade visual com correção (AV c/c) no período préoperatório $\geq 20 / 20$, e 985 olhos $(98, \%) \geq 20 / 40$. No período pós-operatório, 598 olhos $(59,8 \%)$ encontravam-se com acuidade visual sem correção (AV s/c) melhor ou igual a 20/20 e $919(91,9 \%)$ melhor ou igual a 20/40 (Gráfico 1).

A média do EE refracional no período pré-operatório foi de

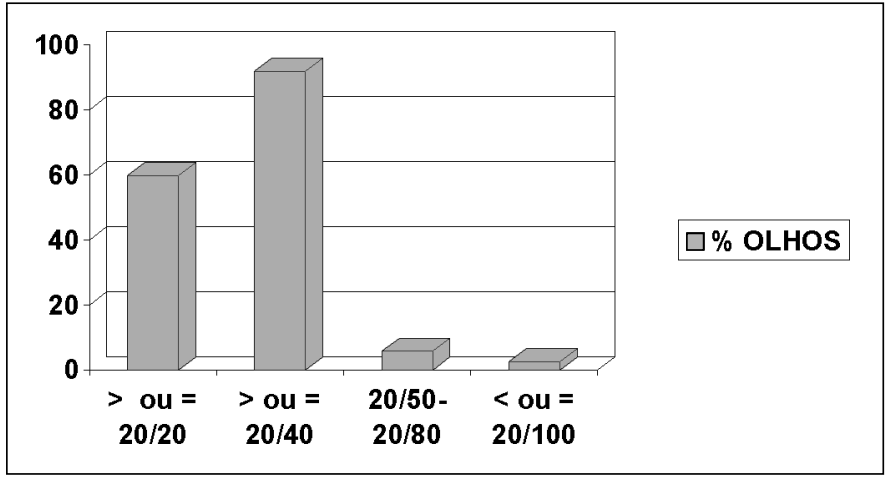

Gráfico 1 - Análise da acuidade visual sem correção no último exame de pacientes submetidos a LASIK $(n=1000)$ 
$-4,29 \pm 3,20 \mathrm{D}$ (variando de $-20,00 \mathrm{a}+3,50 \mathrm{D}$ ) reduzindo para $-0,20 \pm 0,62 \mathrm{D}(-6,62 \mathrm{a}+2,50 \mathrm{D})$ ao último exame pós-operatório. Setecentos e sessenta e sete olhos $(76,7 \%)$ ficaram com EE refracional dentro da faixa de $\pm 0,50 \mathrm{D}$ e 926 olhos $(92,6 \%)$ ficaram com $\pm 1,00 \mathrm{D}$ da emetropia. Somente 25 casos $(2,5 \%)$ ficaram fora da faixa entre $\pm 2,00 \mathrm{D}$.

Houve redução do astigmatismo refracional de $-1,18 \pm$ $1,25 \mathrm{D}(+3,00$ a $-9,00 \mathrm{D})$ para $-0,25 \pm 0,48 \mathrm{D}(+0,75 \mathrm{a}-5,00 \mathrm{D})$ do período pré para o pós-operatório respectivamente.

Dividimos as complicações do LASIK em per-operatórias, e pós-operatórias precoces (até 30 dias de cirurgia) e tardias.

Cinco olhos $(0,5 \%)$ apresentaram complicações no período per-operatório relacionadas ao disco corneano: 3 discos finos, 1 disco com perfuração central ("buttonhole"), e 1 disco pequeno. $\mathrm{O}$ caso com perfuração central do disco apresentava ceratometria pré-operatória de 44,50 x 43,71 D e paquimetria de $554 \mathrm{~mm}$. Destes olhos, 4 foram operados com o microceratótomo $\mathrm{ACS}^{\circledR} \mathrm{e} 1$ com o Hansatome ${ }^{\circledR}$.

No primeiro dia de pós-operatório foi observado discreto edema epitelial em 8 olhos $(0,8 \%)$, com regressão após uso da medicação usual.

No período pós-operatório precoce, a complicação mais freqüente foram dobras no disco (64 olhos ou 6,4\%), sendo que em apenas 4 casos $(0,4 \%)$ foi necessária reintervenção cirúrgica com irrigação da interface e reposicionamento do disco, devido a baixa acuidade visual (Figura 1). Além destes, $3(0,3 \%)$ outros casos apresentaram-se no pós-operatório imediato com o disco deslocado; dois tinham história positiva para trauma ocular. Os discos foram reposicionados como descrito acima, sendo que em 1 caso $(0,1 \%)$ foi necessária a realização de sutura de contenção (Figura 2). Destes 67 olhos com dobras e deslocamento do disco, $40(59,7 \%)$ foram operados com o microceratótomo ACS $^{\circledR}$ e $27(40,29 \%)$ com o Hansatome ${ }^{\circledR}$.

Os depósitos na interface foram a segunda complicação

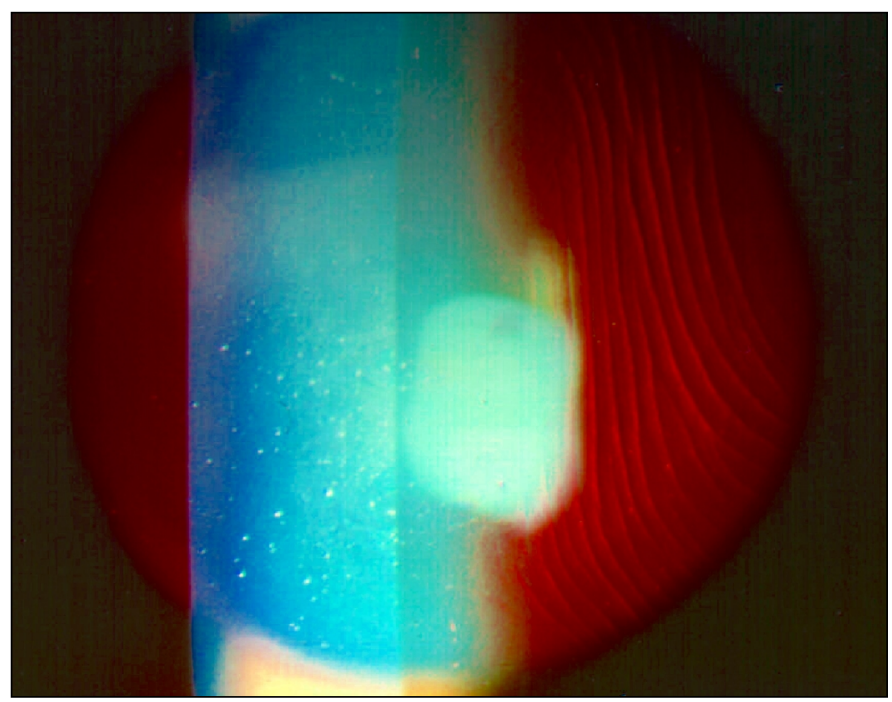

Figura 1 - Dobras de disco em paciente submetido à cirurgia de LASIK

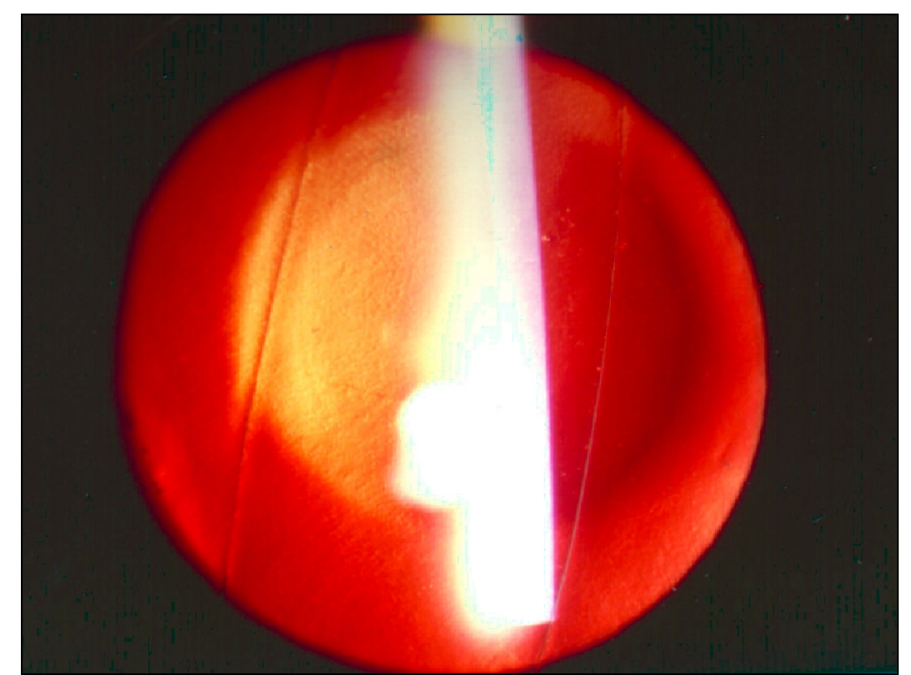

Figura 2 - Sutura de contenção

mais freqüentemente observada, perfazendo um total de 41 casos (4,1\%). Destes, 27 (65,85\%) eram debris inespecíficos, 7 $(17,07 \%)$ eram pequenos filamentos de algodão ou da própria esponja de Merocel $^{\oplus}, 6(14,63 \%)$ eram depósitos hemáticos, e $1(2,43 \%)$ foi classificado como depósito pigmentado. Estes casos não foram submetidos a reintervenção cirúrgica, pois os depósitos mostravam-se inertes e fora do eixo visual.

Em 4 casos $(0,4 \%)$, a partir do exame de 1 mês de pósoperatório, havia a descrição de pequenos infiltrados de localização aparentemente subepitelial, semelhantes às opacidades corneanas observadas no período pós-operatório de PRK, tendo sido classificados como traços de "haze". Três deles haviam sido operados com o microceratótomo Hansato$m \mathrm{e}^{\circledR}$. A regressão espontânea ocorreu entre 2 meses e 1 ano.

Onze casos $(1,1 \%)$ apresentaram-se com queixa de baixa de acuidade visual de intensidade variável e fotofobia. Observamos ao exame a presença de infiltrado estromal, diagnosticado como ceratite não específica da interface ou Síndrome de Areias do Saara ("Sands of Sahara"). Nestes casos, foi modificada a medicação pós-operatória, tendo sido intensificado o esquema de corticoterapia tópica (Acetato de Prednisolona $1 \%$ : Pred Fort ${ }^{\circledR}$ ) até melhora do quadro clínico. Oito olhos $(72,72 \%)$ haviam sido operados com o microceratótomo Hansatome $^{\circledR}$, e $3(27,27 \%)$ com o ACS $^{\circledast}$.

Em um caso $(0,1 \%)$ foi observado infiltrado na interface, de diagnóstico duvidoso, fazendo-se a opção pelo levantamento do disco e coleta de material para pesquisa do agente etiológico. Introduzido antibioticoterapia tópica (Ciprofloxacina $3 \%$ : Ciloxan $^{\circledR}$ ), tendo sido suspensa após resultado negativo dos exames. O diagnóstico presuntivo foi infiltrado estéril.

Um paciente submetido à cirurgia bilateral retornou no $10^{\circ}$ pós-operatório, com queixa de dor ocular unilateral, fotofobia, lacrimejamento e discreto embaçamento visual. Ao exame biomicroscópio, observou-se ceratite epitelial de aspecto semelhante à infecção por herpes simples. Tratado com pomada de 
Acyclovir 3\% $\left(\right.$ Zovirax $^{\circledR}: 5 x /$ dia por 14 dias), com melhora da sintomatologia. Este paciente não apresentava história prévia positiva para herpes ocular.

Um paciente $(\mathrm{n}=2)$ apresentou aumento transitório e discreto da pressão intra-ocular, no $20^{\circ}$ dia de pós-operatório, ainda na vigência de corticoterapia tópica. Foi retirado o corticóide, com retorno aos níveis pressóricos pré-operatórios.

Quatro casos $(0,4 \%)$ apresentaram crescimento epitelial periférico da interface. Em três deles (75\%) foi utilizado o microceratótomo $\mathrm{ACS}^{\circledR}$, não sendo necessária reintervenção cirúrgica em nenhum caso.

As complicações no período intra-operatório e pósoperatório precoce podem ser melhor avaliadas na tabela 1.

Para as complicações pós-operatórias tardias foram considerados apenas os casos com seguimento mínimo de 3 meses ( $\mathrm{n}=655$ ou $65,5 \%)$. O EE pós-operatório médio neste grupo foi de $-0,26 \pm 0,76 \mathrm{D}$ variando de $-6,62 \mathrm{D} \mathrm{a}+2,50 \mathrm{D}(\mathrm{n}=655)$.

Destes, 53 olhos $(8,09 \%)$ ficaram hipocorrigidos acima de $1,00 \mathrm{D}$; sendo que 38 olhos (5,8\%) estavam entre 1,10 e 2,00D; e 15 olhos $(2,29 \%)>2$ D.

Onze casos $(1,67 \%)$ apresentaram hipercorreção superior a $1,00 \mathrm{D}$, sendo que $7(1,06 \%)$ estavam entre 1,10 e $2,00 \mathrm{D}$, e 4 $(0,61 \%)$ acima de $2 \mathrm{D}$.

Dos 655 olhos, 28 (4,27\%) necessitaram de re-tratamento, sendo que 1 apresentava cirurgia intra-ocular prévia (transplante de córnea + facectomia com implante de lente intraocular + incisão relaxante). $\mathrm{O}$ tempo médio para a realização do re-tratamento foi de 10,5 $\pm 9,31$ meses ( 3 a 48 meses). Apenas os míopes $(\mathrm{n}=3)$ e astigmatas (astigmatismo miópico simples, composto ou misto; $n=26$ ) necessitaram de re-tratamento. Em todos eles, fez-se opção pelo levantamento do disco. Dos casos reoperados, $15(53,57 \%)$ apresentavam EE miópico $\leq 6,00 \mathrm{D}$ antes da primeira cirurgia e $13(46,42 \%)>6,00 \mathrm{D}$. O re-tratamento foi indicado em 26 olhos hipocorrigidos $(3,96 \%)$ e em 2 hipercorrigidos $(0,3 \%)$.

Após o re-tratamento, um caso apresentou por duas vezes deslocamento do disco, sendo necessário reposicioná-lo.

A acuidade visual corrigida final manteve-se igual à préoperatória em 515 casos $(78,62 \%)$. As alterações da melhor acuidade visual com correção podem ser melhor analisadas no gráfico 2 .

\begin{tabular}{|c|c|c|}
\hline \multicolumn{3}{|c|}{$\begin{array}{l}\text { Tabela 1. Complicações no período per e pós-operatório } \\
\text { precoce em } 1000 \text { olhos submetidos a LASIK }\end{array}$} \\
\hline \multicolumn{3}{|c|}{ COMPLICAÇÕES EM LASIK } \\
\hline \multirow{3}{*}{$\begin{array}{l}\text { Per-operatórias } \\
\text { (relacionadas ao } \\
\text { microcerátomo) }\end{array}$} & Disco fino & $0,3 \%$ \\
\hline & Disco pequeno & $0,1 \%$ \\
\hline & Perfuração no disco & $0,1 \%$ \\
\hline \multirow{5}{*}{$\begin{array}{l}\text { Pós-operatórias } \\
\text { (precoces) }\end{array}$} & Dobras no disco & $6,4 \%$ \\
\hline & Debris na interface & $4,1 \%$ \\
\hline & Ceratite não específica da interface & $1,1 \%$ \\
\hline & "Haze" & $0,4 \%$ \\
\hline & Crescimento epitelial & $0,4 \%$ \\
\hline
\end{tabular}

Dentre os 5 casos que perderam 2 ou mais linhas da melhor acuidade visual corrigida, $4(80 \%)$ mantiveram $20 / 40$ ou melhor. A média do EE pré-operatório destes 5 olhos era de $-15,6 \mathrm{D} \pm 5,43$, e em 4 olhos a miopia era maior que 12,00D, (variação de -6,12D a -19,87D), reduzindo para -2,80 $\pm 1,61 \mathrm{D}$ ao último exame (-0,25D a -4,25D). A média ceratométrica foi reduzida de $42,89 \pm 1,35 \mathrm{D}(40,88$ a $44,68 \mathrm{D})$ para $33,10 \pm 1,94 \mathrm{D}$ do período pré para o pós-operatório respectivamente. Destes, 2 casos apresentavam dobras periféricas do disco que não foram corrigidas cirurgicamente.

Não foram observados casos de ectasia de córnea ou ilha central.

\section{DISCUSSÃO}

A técnica de LASIK, por apresentar um curso pósoperatório pouco doloroso e resultados visuais relativamente rápidos, vem sendo muito bem aceita no meio oftalmológico. Entretanto, ainda apresenta limitações e complicações que devem ser lembradas e estudadas a cada indicação cirúrgica.

Nosso estudo buscou justamente fazer análise crítica das complicações ocorridas em amostra relativamente grande (1000 olhos), operada pelo mesmo cirurgião, que já possuía experiência com cirurgia refrativa lamelar. Desta forma, conseguimos reduzir possíveis variáveis relacionadas às diferenças individuais de técnica cirúrgica ${ }^{(7)}$.

Apesar de uma grande variação no tempo de seguimento (1 a 50 meses), resolvemos estudar todos os casos, pois desta forma teríamos maior amostragem para a análise das complicações per e pós-operatórias precoces.

Para as complicações tardias, relacionadas mais ao resultado refracional final no tocante a hipo e hipercorreções, assim como alterações da melhor acuidade visual corrigida, foram selecionadas apenas os casos com seguimento $\geq 3$ meses, época em que geralmente já apresentamos uma certa estabilidade refracional ${ }^{(7-10)}$.

Vinte e sete olhos haviam sido submetidos à cirurgia ocular prévia. Não observamos complicações neste grupo, à exce-

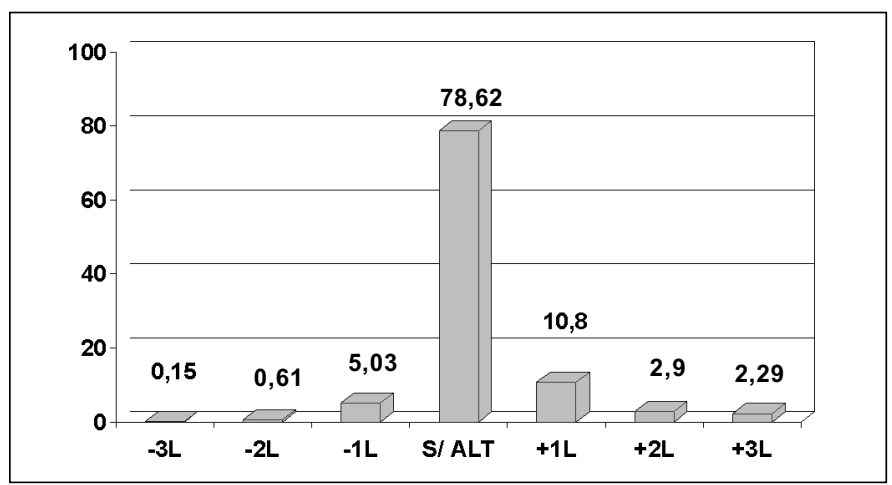

Gráfico 2 - Porcentagem de olhos com alteração da melhor acuidade visual corrigida em relação ao período pré-operatório (Tabela de Snellen) $(n=655)$ 
ção de um paciente transplantado e pseudofácico que necessitou de retoque por hipocorreção. Entretanto cuidados especiais devem ser tomados, uma vez que a literatura nos demonstra uma maior chance de complicações nestes olhos, como por exemplo, crescimento epitelial da interface, principalmente naqueles casos com ceratotomia radial prévia ${ }^{(11)}$.

Apesar de termos tratado pacientes com alta miopia, não foram observadas alterações retinianas, como descolamentos ou membranas neovasculares, que levassem a diminuição da acuidade visual no período pós-operatório. Quatro dos nossos casos tiveram que ser submetidos a fotocoagulação profilática por laser de argônio, devido a degenerações prévias, fato que deve ter colaborado para a redução deste tipo de complicação. Devemos salientar a importância da avaliação pré-operatória da retina, por meio do exame de mapeamento com análise de periferia, como orientado por Arevalo et al. ${ }^{(12)}$. Neste estudo eles relataram a incidência de alterações vítreoretinianas em 29.916 olhos submetidos à técnica de LASIK. Destes, 20 olhos $(0,06 \%)$ apresentaram algum tipo de alteração: 14 evoluíram com descolamento de retina regmatogênico; 4 desenvolveram roturas de retina sem descolamento, 1 apresentou hemorragia vítrea seguida de descolamento de retina após perfuração córneo-escleral durante a realização da ceratectomia, e o último, membrana neovascular justa-foveal.

A técnica de LASIK mostrou-se, de maneira geral, eficaz para a correção dos erros refracionais neste grupo, uma vez que $91,9 \%$ dos casos atingiram acuidade visual sem correção melhor ou igual a 20/40. A previsibilidade pode ser observada pela porcentagem de olhos que ficaram com equivalente esférico refracional dentro da faixa de $\pm 1,00 \mathrm{D}(92,6 \%)$.

Neste estudo as complicações no período per-operatório foram relacionadas à ceratectomia ( 3 discos finos, 1 disco com perfuração central e 1 disco de diâmetro reduzido). Alguns autores atribuem este tipo de complicação a vácuo insuficiente durante o corte, sendo que casos de perfuração central do disco ainda apresentariam maior relação com ceratometrias pré-operatórias muito elevadas ${ }^{(13)}$ olhos com cirurgia ocular prévia $^{(14)}$, ou ainda mau funcionamento do microceratótomo em córneas com curvatura normal ${ }^{(15)}$. Em nosso estudo, este tipo de complicação esteve mais relacionada ao microceratótomo horizontal $\mathrm{ACS}^{\oplus}$ (utilizado em 4 dos 5 casos), dado este concordante com a literatura. Segundo Walker et al., complicações intra-operatórias com o disco ocorreram em $6,7 \%$ dos casos operados com $\mathrm{ACS}^{\circledR}$, e somente $0,3 \%$ com o Hansato$m \mathrm{e}^{\circledast(16)}$. Isto pode ser parcialmente explicado por oscilações da pressão intra-ocular causadas por blefaroespasmos dos pacientes durante $\mathrm{o}$ ato cirúrgico. Aparentemente, $\mathrm{o} \mathrm{ACS}^{\oplus}$ parece ser mais vulnerável a este tipo de oscilação, uma vez que o Hansatome ${ }^{\circledast}$ apresenta mecanismo no qual frente à perda do vácuo há bloqueio do corte ${ }^{(17)}$. Em nenhum caso foi necessária a suspensão do procedimento. Mesmo no caso de perfuração do disco prosseguiu-se com a ablação, pois o erro refracional era baixo, e o orifício pequeno, tendo sido realizado o tratamento no leito após remoção epitelial da área acometida. Este caso apresentou boa evolução. Devemos lembrar que nesta situação, existe maior chance de complicações, uma vez que a área desepitelizada tratada funciona como PRK, o que pode levar ao aparecimento de opacidade corneana local ("haze"), indução de astigmatismo e perda da melhor acuidade visual corrigida. Alguns autores aconselham abortar o procedimento e reintervir após 3 meses fazendo um novo disco ou mudando o procedimento para $\mathrm{PRK}^{(15-16)}$

O edema epitelial observado no primeiro dia foi atribuído à manipulação pré-operatória, uma vez que houve regressão com uso da medicação usual.

As dobras do disco foram, entre as complicações precoces, as mais freqüentes. Embora pouco percebidas sob o microscópio ao final do ato cirúrgico, são notadas no período pós-operatório ao exame de lâmpada de fenda ${ }^{(10)}$. Estas dobras, de acordo com a sua intensidade e localização, podem levar a astigmatismos irregulares, perda de linhas da melhor acuidade visual corrigida ${ }^{(10,18)}$, distorção da imagem e diplopia $^{(18)}$. Carpel et al. ${ }^{(19)}$ classificaram as estrias ou dobras em cinco tipos, de acordo com a localização (superficial ou profunda) e intensidade, direcionando com isso o tratamento e o prognóstico visual. Em nosso estudo, tivemos 64 olhos $(6,4 \%)$ com dobras no disco, sendo que destes, apenas $4(0,4 \%)$ necessitaram de reintervenção cirúrgica para regularização da superfície. Nos demais casos, optou-se pela observação, por não estarem interferindo na acuidade visual ou por estarem localizadas na periferia. A freqüência de dobras no disco na literatura é bastante variável e encontra-se entre 0 a $66,64 \%$ havendo concordância em todos, quanto à indicação de reposicionamento do disco nos casos com baixa de acuidade visual $^{(9-10,20-23)}$. Outros autores descrevem técnicas para evitar a formação de dobras, como Pannu et al. ${ }^{(20)}$ que utilizam, de rotina lentes de contato terapêuticas no pós-operatório imediato até 30 minutos, quando então o paciente é reavaliado. Se constatado um bom posicionamento do disco, a lente é retirada, e o paciente liberado.

Por outro lado, os deslocamentos do disco dificilmente ocorrem espontaneamente. Em nossa série, 3 casos $(0,3 \%)$ apresentaram-se com o disco deslocado, sendo que em 2 havia história de traumatismo. Alguns autores descrevem o deslocamento de disco como complicação rara e séria, que pode levar a crescimento epitelial na interface, dobras e haze ${ }^{(7,10)}$. Portanto, é de extrema importância a orientação do paciente quanto à fragilidade da adesão disco-estroma no pós-operatório precoce, de forma a tentar reduzir este tipo de complicação, que pode ocasionar baixa de acuidade visual permanente $^{(7)}$. Nos deslocamentos, torna-se obrigatória a reposição do disco, após retirada de epitélio do leito estromal, sendo às vezes, necessária a realização de suturas de contenção ${ }^{(7,13)}$.

As dobras e deslocamentos parecem ter relação direta não só com o tipo de microceratótomo utilizado, mas também com a experiência do cirurgião em evitar irrigação excessiva do estroma ${ }^{(7)}$ e em remover o blefarostato do paciente após constatar a perfeita adesão do disco. Dos 67 olhos que apresen- 
taram dobras ou deslocamento, $59,7 \%$ foram operados com o microceratótomo $\mathrm{ACS}^{\circledR}$.

Depósitos na interface são também descritos na literatura, com incidência variando de 2,6 a 40\% ${ }^{(24-25)}$. Os depósitos hemáticos são geralmente mais observados em uma ou mais das seguintes situações: pacientes que eram usuários de lentes de contato, e desenvolveram neovascularização corneana periférica; em casos de córneas com diâmetros reduzidos, ou quando se utiliza microceratótomos com discos de grande diâmetro (cerca de 9,5mm) ${ }^{(21)}$. Os depósitos podem ainda estar inversamente relacionados à habilidade do cirurgião e cuidados durante a lavagem copiosa do leito estromal ablado(6, 8-10, 22). Aqui, assim como já discutido, a iluminação do microscópio cirúrgico nem sempre possibilita o diagnóstico da presença destes depósitos. Desta forma, o exame em lâmpada de fenda logo após o procedimento pode ser de grande valia, para reintervenção imediata, caso se julgue necessário ${ }^{(8)}$ embora na grande maioria das vezes esses depósitos não alterem a acuidade visual ${ }^{(6,18,23)}$. Quando o diagnóstico é feito no primeiro dia do período pós-operatório, devemos levar em consideração o tipo e tamanho do depósito, a localização (se no eixo visual), e a presença ou não de reação inflamatória local, para decidirmos sobre a conduta a ser tomada.

Alguns autores já descreveram a presença de "haze" no pós-operatório de LASIK, geralmente em menor intensidade do que o relatado após cirurgia de PRK, e com regressão em torno do primeiro ao terceiro mês de cirurgia ${ }^{(3,7,10,23,26)}$. Nossa incidência foi de apenas $0,4 \%$, sendo todos os casos classificados como traços de "haze", evoluindo também com regressão espontânea, sem perda da melhor acuidade visual corrigida. Não observamos correlação positiva entre a presença do "haze" e problemas intra-operatórios com o disco (discos mais finos ou com perfuração central).

Onze de nossos casos $(1,1 \%)$ apresentaram-se com fotofobia e baixa de acuidade visual associada a infiltrado estromal, tendo sido diagnosticado como ceratite não específica da interface. Esta é uma complicação do LASIK descrita mais recentemente ${ }^{(27)}$. Manifesta-se geralmente entre o primeiro e quinto dia após a cirurgia, através de infiltrado com aspecto de areia peneirada, na interface do estroma com o disco corneano, aumentando em extensão e densidade progressivamente antes da sua resolução ${ }^{(28)}$. Sua causa continua desconheci$\mathrm{da}^{(27-28)}$, podendo estar relacionada a vários fatores desencadeantes. Andrade et al. ${ }^{(27)}$ relataram a importância do diagnóstico correto e precoce nos pacientes com "Sands of Sahara", intensificando a corticoterapia tópica, o que contribuiu para a atenuação das seqüelas relacionadas a este processo inflamatório. Holland et al. ${ }^{(28)}$ realizaram investigação epidemiológica nos seus casos de ceratite inespecífica da interface. Eles postularam a liberação de toxina de flora Gram negativa, presente na água destilada armazenada em autoclaves. Esta endotoxina pode sobreviver à esterilização com ciclos curtos de vapor, sendo então introduzida na interface durante a ceratectomia, incitando reação imunológica com presença de polimorfonucleares em indivíduos suscetíveis.
O diagnóstico diferencial da ceratite não específica da interface é extremamente importante, principalmente no que se refere a processos infecciosos. Na dúvida, deve-se optar pelo levantamento do disco e coleta de material para esclarecimento diagnóstico ${ }^{(29-32)}$. Um dos nossos casos que cursou com infiltrado estromal de diagnóstico duvidoso foi submetido à coleta do material, mas o resultado foi negativo, tendo sido considerado o infiltrado como estéril.

Um dos nossos casos retornou sintomaticamente em torno do $10^{\circ}$ dia pós-operatório, sendo diagnosticado quadro de ceratite epitelial herpética, apesar de não ter história positiva. O trauma cirúrgico da dissecção lamelar ou a luz ultravioleta do excimer laser podem ser responsáveis pela reativação do vírus do herpes. Alguns autores preconizam a utilização de antiviral tópico e sistêmico profilático em pacientes sabidamente portadores do herpes vírus antes da cirurgia refrativa $^{(33)}$.

A hipertensão ocular observada após a cirurgia não foi complicação freqüentemente observada. $\mathrm{O}$ único paciente $(n=2)$ que desenvolveu aumento de seus níveis pressóricos continuava por conta própria com esquema de corticoterapia tópica no $20^{\circ}$ dia de pós-operatório. O controle ocorreu após a suspensão da droga.

$\mathrm{O}$ crescimento epitelial na interface geralmente encontrase associado a irregularidade nas bordas do disco e leito estromal, sendo mais freqüente nas regiões inferior e tempo$\mathrm{ral}^{(10)}$. Cuidados com o bom posicionamento do disco, irrigação e limpeza do estroma ablado com esponja de Merocel $^{\circledast}$ reduzem significantemente esta complicação. Caso o crescimento ocorra na córnea periférica, não é indicada sua remoção cirúrgica $^{(9,13,34)}$. Perez-Santoja et al. ${ }^{(10)}$ relataram "melting" (lise) do disco corneano em 3,5\% a 5,7\%, secundário a áreas de grande crescimento epitelial na interface. Quando julgado necessário, o disco deve ser levantado e lavado para retirada do epitélio. Kapadia et al. ${ }^{(35)}$ propuseram PRK para tratamento nos casos onde é impossível levantar novamente o disco.

A análise das complicações refracionais foram feitas com base nos dados de 655 olhos com seguimento mínimo de 3 meses. Hipocorreção superior a $1,00 \mathrm{D}$ foi a mais freqüentemente observada, em 8,09\% dos casos. Resultados semelhantes foram observados no estudo de Salah et al. ${ }^{(36)}$ onde no $5^{\circ}$ mês após a cirurgia, $11,4 \%$ dos casos apresentavam-se hipocorrigidos acima de 1,00D. Kwitko et al ${ }^{(9)}$ descreveram a incidência de hipocorreção acima de 1,00D variando de $5,5 \%$ a $40 \%$, de acordo com a ametropia pré-operatória.

A incidência de hipercorreção na literatura também é bastante variável, oscilando de $3,7 \%$ a $20 \%{ }^{(6,9,10,36)}$. Em nosso estudo, a incidência foi menor, em torno de 1,67\%.

Os re-tratamentos foram necessários em 28 olhos (4,27\%) levando a melhora da acuidade visual final sem correção em todos os casos. A grande maioria destes casos encontrava-se hipocorrigida ( $\mathrm{n}=26$ ou $3,96 \%$ ). Esperou-se período mínimo de 3 meses para se indicar a reoperação. Alguns autores consideram este tempo como suficiente para estabilidade refracional 
nos casos com miopia pré-operatória entre 8,00 e 16,00D, devendo-se aguardar pelo menos 6 meses nas miopias entre 16,00 e $20,00 \mathrm{D}^{(10)}$. A incidência de reoperações na literatura varia de 0 a $17 \%$, de acordo com a ametropia pré-operatória, sendo os nossos resultados discordantes a princípio, onde a porcentagem de reoperação foi muito semelhante em ametropias inferiores e superiores a $-6,00 \mathrm{D}^{(10,23,26,36-39)}$.

Perda da melhor acuidade visual corrigida pode ocorrer por inúmeras causas, entre elas, ilha central, descentração da ablação ou zona óptica pequena, dobras centrais no disco, astigmatismos irregulares ou alterações da topografia cornea$\mathrm{na}^{(6,8-10,22-23)}$. Sua incidência varia de $0 \%$ a $33,3 \%{ }^{(22,37)}$. Em nosso estudo, perda igual ou superior a 2 linhas foi observada em apenas 5 casos $(0,76 \%)$, sendo que o aplanamento médio destas córneas foi de 9,79D em relação ao pré-operatório. O aplanamento corneano pós-operatório acentuado pode ter contribuído para esta alteração da acuidade visual corrigida, visto que não foram observadas outras complicações que justificassem a baixa visual, exceto dobras de disco fora do eixo visual em 2 casos.

Em nosso estudo, não observamos complicações graves que pudessem levar a perda definitiva da acuidade visual de nossos pacientes, como ectasia de córnea ou perfuração ocular. As poucas complicações anteriormente descritas puderam, em sua grande maioria, ser prontamente resolvidas.

Em resumo, acreditamos que a técnica de LASIK pode ser considerada procedimento refrativo seguro, desde que sejam respeitados alguns fatores na avaliação pré-operatória. As complicações associadas a este procedimento são pouco freqüentes, estando diretamente relacionadas à experiência do cirurgião e qualidade dos equipamentos utilizados tanto para a realização da ceratectomia quanto para a fotoablação.

\section{A B S T R A C T}

Purpose: To analyze the incidence, type, management and evolution of complications in laser in situ keratomileusis (LASIK). Methods: Retrospective non-comparative case series. LASIK was performed in 1000 eyes using the Hansato$\mathrm{me}^{\circledR}$ or the Automated Corneal Shaper ${ }^{\circledR}$ microkeratomes, and the VISX 20/20B or the Chiron Technolas 217C excimer lasers. Intraoperative, early and late postoperative complications were reviewed. Results: Mean preoperative spherical equivalent (SE) was $-4.29 \pm 3.20 \mathrm{D}$. Average follow-up was $6.05 \pm$ 6.69 months. There were $5(0.5 \%)$ intraoperative microkeratome-related flap complications ( 3 thin flaps, 1 buttonhole and 1 small flap). The most frequent early postoperative complication was microwrinkled flaps $(6.4 \%)$, followed by interface debris $(4.1 \%)$, the non-diffuse intralamellar keratitis $(1.1 \%)$, haze $(0.4 \%)$, interface epithelial ingrowth $(0.4 \%)$ and dislocated flaps $(0.3 \%)$. Most of these reported events were successfully managed. Late refraction-related complications were analyzed in 655 eyes with a minimum follow-up of 3 months. At the last examination, mean SE was $-0.26 \pm 0.76 \mathrm{D}$. Eight per cent were undercorrected by more than $-1.00 \mathrm{D}$ and $1.67 \%$ was overcorrected by more than $+1.00 \mathrm{D}$. Reoperation was necessary in 28 eyes $(4.27 \%)$. Five cases $(0.76 \%)$ lost 2 or more lines of best spectacle corrected visual acuity. There were no sight-threatening complications. Conclusion: LASIK is a safe refractive procedure with few complications.

Keywords: Laser in situ keratomileusis/adverse effects; Laser in situ keratomileusis/methods; Refractive errors/surgery; Prospective studies; Intraoperative complications/epidemiology; Postoperative complications/epidemiology

\section{REFERÊNCIAS}

1. Trokel SL, Srinivasan R, Braren B. Excimer laser surgery of the cornea. Am J Ophthalmol 1983;96:710-5.

2. McDonald MB, Liu JC, Byrd TJ, Abdelmegeed M, Andrade HA, Klyce SD, Varnell et al. Central photorefractive keratectomy for myopia: partially sighted and normally sighted eyes. Ophthalmology 1991;98:1327-37.

3. Pallikaris IG, Papatizanaki ME, Siganos DS, Tsilimbares MK. A corneal flap technique for laser in situ keratomileusis. Human studies. Arch Ophthalmol 1991;109:1699-702.

4. Forseto AS, Nosé RAM, Nosé W. PRK versus LASIK para correção de miopia baixa e moderada. Arq Bras Oftalmol 2000;63:257-62.

5. Pirzada WA, Kalaawry H. Laser in situ keratomileusis for myopia of -1 to -3.50 diopters. J Refract Surg 1997;13(5 Suppl):425-6.

6. Mori ES, Suzuki CK, Allemann N, Schor P, Campos M, Chamon W. Ceratectomia fotorrefrativa associada a ceratectomia lamelar pediculada (LASIK) para correção de miopia e astigmatismo moderados e altos em um serviço universitário. Arq Bras Oftalmol 1998;61:585-9.

7. El-Maghraby A, Salah T, Waring III GO, Klyce S, Ibrahim O. Randomized bilateral comparison of excimer laser in situ keratomileusis and photorefractive keratectomy for 2.50 to 8.00 diopters of myopia. Ophthalmology 1999;106: 447-57.

8. Rashad KM. Laser in situ keratomileusis for myopic astigmatism. J Refract Surg 1999; 15:653-60.

9. Kwitco S, Marinho D, Raskin R, Sprinz S, Rabin M, Rymer S, Noble AM. LASIK para correção de miopia, astigmatismo e hipermetropia. Arq Bras Oftalmol 2000;63:9-17.

10. Pérz-Santoja JJ, Bellot J, Claramonte P, Ismail MM, Alió JL. Laser in situ keratomileusis to correct high myopia. J Cataract Refract Surg 1997;23:372-85.

11. Forseto AS, Nosé RAM, Francescone CM, Nosé W. Laser in situ keratomileusis for undercorrection after radial keratotomy. J Refract Surg 1999;15: 424-8.

12. Arevalo JF, Ramirez E, Suarez E, Moralles-Stopello J, Cortez R, Ramirez J, et al. Incidence of vitreoretinal pathologic conditions within 24 months after laser in situ keratomileusis. Ophthalmol 2000;107:258-62.

13. Gimbel HV, Penno EEA, Van Westenbrugge JA, Ferenzowicz M, Furlong MT. Incidence and management of intraoperative and postoperative complications in 1000 consecutive laser in situ keratomileusis cases. [commented on Ophthalmology 1999;106:1455-7] Ophthalmology 1998;105:1839-48.

14. Stulting RD, Carr JD, Thompson KP, Waring III GO, Wiley WM, Walker JG. Complications of laser in situ keratomileusis for the correction of myopia. Ophthalmology 1999;106:13-20.

15. Leung AT, Rao SK, Cheng AC, Yu EW, Fan DS, Lam DS. Pathogenesis and management of laser in situ keratomileusis flap buttonhole. J Cataract Refract Surg 2000;26:358-62.

16. Walker MB, Wilson SE. Lower intraoperative flap complication rate with the Hansatome microketome compared to Automated Corneal Shaper. J Refract Surg 2000;16:79-82.

17. Burato L. Down-up Lasik with the Hansatome: personal technique. In: Burato L, Brint S, editors. Lasik surgical techniques and complications. 2rd.ed. Detroit: Slack Thorafare; 1998. p. 131-64.

18. Holland SP, Srivannaboon S, Reinstein DZ. Avoiding serious corneal complications laser assisted in situ keratomileusis and photorefractive keratectomy. Ophthalmology 2000;107:640-52. 
19. Carpel EF, Carlson KH, Shannon S. Folds and striae in laser in situ keratomileusis flaps. J Refract Surg 1999;15:687-90.

20. Pannu JS, Mutyala S. Corneal flap adhesion following LASIK. J Cataract Refract Surg 1999;25:606.

21. Velasco-Martinelli EJ, Tarcha FA. Superior hinge laser in situ keratomileusis. J Refract Surg 1999;15(2 Suppl):S209-11.

22. Carvalho M, Nascimento E, Chamon W, Allemann N, Campos M, Scarpi MJ. LASIK na correção da alta miopia. Arq Bras Oftalmol 1997;60:570-5.

23. Condon PI, Mulhern M, Fulcher T, Foley- Nolan A,O' Keef M. Laser intrastromal keratomileusis for high myopia and myopic astigmatism. Br J Ophthalmol 1997;81:199-206.

24. Burato L, Ferrari M, Genisi C. Keratomileusis for myopia with the excimer laser (Burato technique): short term results. J Refract Corneal Surg 1993; 9(suppl): S130-3.

25. Kremer I, Blumenthal M. Myopic Keratomileusis in situ combined with VISX 20/20 photorrefrative keratectomy. J Cataract Refract Surg 1995;21:508-11.

26. Knorz MC, Liermann A, Seiberth V, Steiner H, Wiesknger B. Laser in situ keratomileusis to correct myopia of $-6,00$ to $-29,00$ diopters. J Refract Surg 1996; $12: 575-84$.

27. Andrade BBA, Forseto AS, Francesconi CM, Alves MR, Nosé W. Ceratite não específica da interface pós-laser ceratomileusis-lasik. Rev Bras Oftalmol 2000;59:322-7.

28. Holland SP, Mathias RG, Morck DW, Chiu J, Slade SG. Diffuse Lamellar Keratitis Related to Endotoxins Released from Sterilizer Reservoir Biofilms. Ophthalmol 2000;107:1227-34.
29. Webber SK, Lawless MA, Sutton GL, Rogers CM. Staphylococcal infection under a LASIK flap. Cornea 1999;18:361-5.

30. Kim HM, Song JS, Han HS, Jung HR. Streptococcal keratitis after myopic laser in situ keratomileusis. Korean J Ophthalmol 1998;12:108-11.

31. Haw WW, Manche EE. Sterile peripheral keratitis following laser in situ keratomileusis. J Refract Surg 1999;15:61-3.

32. Al-Reefy M. Bacterial keratitis following laser in situ keratomileusis for hiperopia. J Refract Surg 1999;15(2 suppl):216-7.

33. Davidorf JM. Herpes Simplex keratitis after LASIK.[letter] J Refract Surg 1998; 14:667.

34. Filatov V, Vidaurri-Leal JS, Talamo JH. Selected complications of radial keratomy, photorefractive keratectomy, and laser in situ keratomileusis. Int Ophthalmol Clin 1997;37:123-48.

35. Kapadia MS, Wilson SE. Transepithelial photorefractive keratectomy for treatment of thin flaps or caps after complicated laser in situ keratomileusis. Am J Ophtalmol 1998;126:827-9.

36. Salah T, Waring III GO, El- Maghraby A, Moadel K, Grimm SB. Excimer laser in situ keratomileusis under a corneal flap for myopia of 2 to 20 diopters. Am J Ophthalmol 1996;121:143-55.

37. Güel JL, Muller A. Laser in situ keratomileusis (LASIK) for myopia from -7 to -18 diopters. J Refract Surg 1996;12:222-8.

38. Marinho A, Pinto MC, Pinto R, Vaz, F, Neves MC. LASIK for high myopia: one year experience. Ophthalmic Surg Lasers 1996;27(5 Suppl):S517-20.

39. Gimbel HV, Basti S, Kayne GB, Ferensowicz M. Experience during the learning curve of laser in situ keratomileusis. [commented on J Cataract Refract Surg 1996;22:513-4]. J Cataract Refract Surg 1996;22:542-50.

\section{ABO ELETRÔNICO}

\section{A versão eletrônica dos Arquivos Brasileiros de} Oftalmologia com textos completos está disponível em:

- http://www.abonet.com.br

- SciELO - Scientific Electronic Library Online -

http://www.scielo.org

- http://www.freemedicaljournals.com 